\title{
Nonlinear Modal Analysis of Mechanical Systems with Frictionless Contact Interfaces
}

\author{
Denis Laxalde, Mathias Legrand and Christophe Pierre \\ Structural Dynamics and Vibration Laboratory \\ Department of Mechanical Engineering \\ McGill University, Montreal, QC, Canada
}

\begin{abstract}
This paper investigates the nonlinear analysis, in the form of nonlinear modes, of mechanical systems undergoing unilateral frictionless contact. The nonlinear eigenproblem is introduced by a Rayleigh quotient minimization with inequality constraints formulated in the frequency domain. An augmented Lagrangian procedure is used for the calculation of the nonlinear contact forces. The efficiency of the proposed method for large-scale mechanical systems involving non-smooth nonlinear terms is shown. An industrial application consisting of a compressor blade in contact with a rigid casing is proposed. Sensitivity of the nonlinear modal parameters to contact is illustrated.
\end{abstract}

\section{Introduction}

The concept of nonlinear modes is now commonly accepted as a relevant approach for the analysis of nonlinear dynamical systems [1. While the variety of analytical methods developed during the past decades have contributed to strengthen the theoretical background on nonlinear modes, new challenges appear when it comes to applying this tool to large scale systems [2. An encouraging perspective is however brought by numerical methods such as invariant manifold approach [3], asymptotic-numerical methods [4, shooting and continuation [5] or Fourier methods [6].

Dealing with non-smooth nonlinearities yields other challenges both on numerical analysis and phenomenological aspects. Concerning contact nonlinearities, difficulties come from their essential nonsmooth nature. As contact is defined by a multi-valued law which takes the form of inequality constraints, solution methods are often related to optimization [7, 8]. Dual formulations then provide physical sense to Lagrange multipliers. When it comes to numerical formulations, a popular and robust approach to deal with contact constraints is to introduce augmented Lagrangian functionals [9 11.

In contact dynamics, most integrated approaches are based on time integration and alternatives are unfortunately seldom explored [12,13. Thus, considering analysis of contact dynamics in the framework of nonlinear modes seems an interesting yet challenging approach. In this paper, we investigate the dynamics of linear elastic and autonomous flexible structures with contact interfaces. The target application concerns rotating components in turbomachinery which can be subject to intermittent contacts (or impacts) with surrounding stationary structures. Complex phenomena often occurs in such applications [14 16] and a modal approach appears interesting in order to describe their essential nature in particular.

The proposed approach is first described in a continuum mechanics formalism in which the problem of finding eigensolutions can be regarded as a mixed frequency-time boundary value problem. The solution is sought in the form of a Fourier series and the eigenvalue problem reduces to finding critical values of a generalized Rayleigh quotient. An inequality constrained optimization problem then arises which is solved using an augmented Lagrangian approach. Then, approximations for numerical treatments and algorithm are described.

A numerical application is proposed in which the first nonlinear mode of a turbomachinery compressor blade with contact occurring at its tip edge is explored. Effects of contact on modal parameters are investigated.

\footnotetext{
*Address all correspondence to this author.
} 


\section{Theoretical formulation}

A flexible structure in intermittent contact with a rigid foundation is investigated. Since attention is given to eigensolutions of the nonlinear problem [1, no external forces are applied such as the final system of equations is autonomous. Usual linear damping is neglected as well as friction.

Two main difficulties arise. First, the non-differentiability of the non-smooth contact conditions with respect to the displacement field, makes any linearization impossible. Second, as in every modal formulation, displacements and frequency of the motion are to be determined.

\subsection{Unilateral frictionless contact formulation}

A flexible component in contact with a rigid foundation is considered as illustrated in Fig. 1. Contact

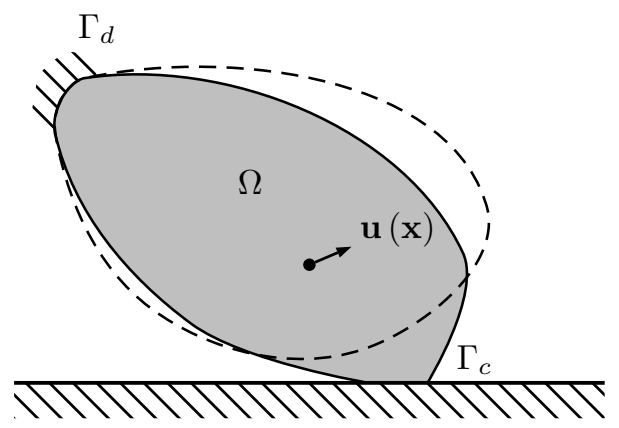

Figure 1: Notations for contact formulation

forces acting on interface $\Gamma_{c}$ are defined according to a continuous gap function separating the possibly contacting bodies:

$$
\mathbf{g}(\mathbf{u})=\mathbf{u}(\mathbf{x}) \cdot \mathbf{n}-\mathbf{g}_{0}(\mathbf{x})
$$

where $g_{0}(\mathbf{x})$ represents the initial positive gap, $\mathbf{n}$, the outward normal to $\Gamma_{c}$, and $\mathbf{u}=\left(u_{1}, u_{2}, u_{3}\right)$ the displacement field of a material point $\mathbf{x}=\left(x_{1}, x_{2}, x_{3}\right)$. Contact conditions [17, referred to as KuhnTucker optimality conditions are such that for all $\mathrm{x} \in \Gamma_{c}$ :

$$
\tau_{N} \leq 0, \mathbf{g}(\mathbf{u}) \leq 0, \mathbf{g}(\mathbf{u}) \cdot \tau_{N}=0
$$

where $\tau_{N}=\sigma \cdot \mathbf{n}$ stands for the contact pressure acting on $\Gamma_{c}$.

\subsection{Boundary value problem}

The linearized strain tensor mapping displacements to deformations is defined as $\epsilon_{k l}=\frac{1}{2}\left(\partial u_{k} / \partial x_{l}+\partial u_{l} / \partial x_{k}\right)$ and the constitutive linear Hooke law, as $\sigma_{i j}=\lambda \delta_{i j} \epsilon_{k k}+2 \mu \epsilon_{i j}$. The strong formulation of the problem with Dirichlet and Signorini boundary conditions takes the following form:

$$
\begin{aligned}
& \rho \ddot{\mathbf{u}}-\operatorname{div} \boldsymbol{\sigma}(\mathbf{u})=0 \quad \text { on } \Omega \times \mathbb{R}_{*}^{+} \\
& \mathbf{u}=0 \quad \text { on } \quad \Gamma_{d} \times \mathbb{R}_{*}^{+} \\
& \mathbf{g}(\mathbf{u}) \leq 0, \quad \tau_{N} \leq 0 \quad \text { and } \quad \mathbf{g}(\mathbf{u}) \cdot \tau_{N}=0 \quad \text { on } \Gamma_{c} \times \mathbb{R}_{*}^{+}
\end{aligned}
$$

Since the subsequent developments involve variational forms, we introduce the space of admissible displacement fields:

$$
V=\left\{\mathbf{u}=\left(u_{1}, u_{2}, u_{3}\right), u_{i} \in H^{1}(\Omega), \mathbf{u}=0 \text { on } \Gamma_{d}\right\}
$$

equipped with a classical inner product (denoted $\langle.,$.$\rangle in the following), and the usual linear forms on$ $V \times V$ :

$$
\begin{gathered}
\mathrm{m}(\mathbf{u}, \mathbf{v})=\int_{\Omega} \rho \mathbf{u v} \mathrm{d} \mathbf{x} \\
\mathrm{k}(\mathbf{u}, \mathbf{v})=\int_{\Omega} \boldsymbol{\sigma}(\mathbf{u}): \boldsymbol{\epsilon}(\mathbf{v}) \mathrm{d} \mathbf{x}
\end{gathered}
$$




\subsection{Mixed frequency-time eigenvalue problem}

We are interested in finding periodic and non-trivial solutions of problem (3). As periodic but not necessarily, it seems natural to expand the displacement field in the frequency-domain using Fourier series:

$$
\mathbf{u}(t)=\sum_{n \in \mathbb{Z}} \hat{\mathbf{u}}_{n} e^{\mathrm{j} n \omega t} \text { with } \hat{\mathbf{u}}_{n}=\frac{1}{T} \int_{T} \mathbf{u}(t) e^{-\mathrm{j} n \omega t} \mathrm{~d} t
$$

in which $\omega=2 \pi / T$ is the fundamental eigenfrequency of the motion to be determined.

The eigenvalue problem consists in finding $\{\omega, \hat{\mathbf{u}}\}$, with $\hat{\mathbf{u}}=\left\{\hat{\mathbf{u}}_{n}, n \in \mathbb{Z}\right\}$ such as,

$$
\begin{aligned}
-\operatorname{div} \boldsymbol{\sigma}\left(\hat{\mathbf{u}}_{n}\right)=(n \omega)^{2} \rho \hat{\mathbf{u}}_{n} & \text { on } \Omega \times \mathbb{Z} \\
\hat{\mathbf{u}}_{n}=0 & \text { on } \Gamma_{d} \times \mathbb{Z} \\
\mathbf{g}(\hat{\mathbf{u}}) \leq 0, \quad \tau_{N} \leq 0 \text { and } \quad \mathbf{g}(\hat{\mathbf{u}}) \cdot \tau_{N}=0 & \text { on } \Gamma_{c} \times[0, T]
\end{aligned}
$$

in which the gap function is now expressed using Eq. (6) as:

$$
\mathbf{g}(\hat{\mathbf{u}})=\sum_{n \in \mathbb{Z}} \hat{\mathbf{u}}_{n} \cdot \mathbf{n} e^{\mathrm{j} n \omega t}-\mathbf{g}_{0}
$$

Note that while Eqs. (7a) and (7b) are formulated using frequency-domain fields, Eq. (7c) involves time-domain fields only. Thus Problem (7) can be regarded as a mixed time-frequency domains boundary value problem.

\subsection{Rayleigh quotient and optimization}

Based on the previous eigenvalue problem and using the linear forms defined in Eqs. (5), we introduce the following generalized Rayleigh quotient:

$$
\mathrm{r}(\hat{\mathbf{u}})=\sum_{n \in \mathbb{Z}} \frac{\mathrm{k}\left(\hat{\mathbf{u}}_{n}, \hat{\mathbf{u}}_{n}\right)}{\mathrm{m}\left(\hat{\mathbf{u}}_{n}, \hat{\mathbf{u}}_{n}\right)}
$$

An eigenvector $\hat{\mathbf{u}}$ is a critical point of this Rayleigh quotient and the associated eigenvalue is such that $\omega^{2}=\mathrm{r}(\hat{\mathbf{u}})$. Here, due to the Signorini conditions, critical points of the Rayleigh quotient are solutions of a constrained minimization problem:

$$
\min _{\hat{\mathbf{u}}_{n} \in V} \mathrm{r}(\hat{\mathbf{u}}) \quad \text { s.t. } \mathbf{g}(\hat{\mathbf{u}}) \leq 0 \quad \text { on } \quad \Gamma_{c} \quad \forall t \in[0, T]
$$

Optimality conditions for this constrained minimization problem imply that there exists a Lagrange multiplier time-function $\lambda(t)>0$ such that:

$$
\begin{aligned}
\nabla_{\hat{\mathbf{u}}_{n}} \mathrm{r}(\hat{\mathbf{u}})+\int_{T}\left\langle\lambda(t), \nabla_{\hat{\mathbf{u}}_{n}} \mathbf{g}(\hat{\mathbf{u}})\right\rangle \mathrm{d} t=0 & \forall n \in \mathbb{Z} \\
\lambda(t)>0 \text { and }\langle\lambda(t), \mathbf{g}(\hat{\mathbf{u}})\rangle=0 & \forall t \in[0, T]
\end{aligned}
$$

The constraint $\mathbf{g}(\mathbf{u}) \leq 0$ has been transferred to the Lagrange multiplier $\lambda(t)$ and the integral in Eq. (11a) can be regarded as a continuous equivalent to traditional weighted sum of individual constraints by the Lagrange multipliers.

Expanding Eqs. (11) leads to a variational formulation:

Find $\left\{\hat{\mathbf{u}}_{n}, n \in \mathbb{Z}\right\}$ such as $\forall \mathbf{v} \in V$

$$
\begin{aligned}
2 \mathrm{~m}\left(\hat{\mathbf{u}}_{n}, \hat{\mathbf{u}}_{n}\right)^{-1}\left(\mathrm{k}\left(\hat{\mathbf{u}}_{n}, \mathbf{v}\right)-n^{2} \omega^{2}(\hat{\mathbf{u}}) \mathrm{m}\left(\hat{\mathbf{u}}_{n}, \mathbf{v}\right)\right)+\int_{T}\langle\boldsymbol{\lambda}, \mathbf{v}\rangle e^{\mathrm{j} n \omega t} \mathrm{~d} t=0 & \forall n \in \mathbb{Z} \\
\boldsymbol{\lambda}>0 \text { and }\langle\boldsymbol{\lambda}, \mathbf{g}(\hat{\mathbf{u}})\rangle=0 & \forall t \in[0, T]
\end{aligned}
$$




\subsection{Augmented Lagrangian}

The technique of augmented Lagrange multipliers converts an initially constrained minimization problem into a saddle point problem for which the addition of penalty terms improves convergence and benefits computationally due to subsequent convex properties. The respective Uzawa-like solution algorithm performs descent on the primal displacement variable and ascent on the dual Lagrange multipliers (coinciding with contact forces when convergence is reached).

For problem (10), an augmented Lagrangian can be defined as [18]:

$$
L_{\kappa}(\hat{\mathbf{u}}, \theta)=\mathrm{r}(\hat{\mathbf{u}})+\frac{1}{2} \int_{T}\|\sqrt{\kappa}(\mathbf{g}(\hat{\mathbf{u}})+\theta)\|_{+}^{2} \mathrm{~d} t
$$

in which $\kappa \theta$ represents a Lagrange multiplier and $\kappa$, a diagonal matrix of positive penalty parameters.

During an iteration along the primal variable, the solution algorithm holds estimated values of dual parameters $\theta$ and $\kappa$ and searches for $\hat{\mathbf{u}}(\theta, \kappa)$ that minimizes the augmented Lagrange functional (13) such as:

$$
\nabla_{\hat{\mathbf{u}}_{n}} L_{\kappa}(\hat{\mathbf{u}}, \theta)=0 \quad \forall n \in \mathbb{Z}
$$

or, equivalently:

Find $\left\{\hat{\mathbf{u}}_{n}, n \in \mathbb{Z}\right\}$ such as $\forall \mathbf{v} \in V$

$$
2 \mathrm{~m}\left(\hat{\mathbf{u}}_{n}, \hat{\mathbf{u}}_{n}\right)^{-1}\left(\mathrm{k}\left(\hat{\mathbf{u}}_{n}, \mathbf{v}\right)-n^{2} \omega^{2}(\hat{\mathbf{u}}) \mathrm{m}\left(\hat{\mathbf{u}}_{n}, \mathbf{v}\right)\right)+\int_{T}\left\langle\kappa(\mathbf{g}(\hat{\mathbf{u}})+\boldsymbol{\theta})_{+}, \mathbf{v}\right\rangle e^{\mathrm{j} n \omega t} \mathrm{~d} t=0
$$

Parameter $\theta$ is then adjusted as follows:

$$
\theta \leftarrow \theta+\max _{\mathbf{x}, t}(\mathbf{g}(\hat{\mathbf{u}}),-\theta)
$$

while the penalty coefficient $\kappa$ can be updated if condition (16) did not improve the constraints.

\subsection{Mode normalization}

Since the frequency of the periodic motion is an unknown of the eigenvalue problem, Eq. (15) has more unknowns than equations. This under-determination can be overcome by conducting a mode normalization through one (since the system is conservative) master coordinate which amplitude defines the modal amplitude and which governs all remaining dependent variables. This amplitude is denoted by $q$ and modal parameters $\hat{\mathbf{u}}_{n}(q)$ and $\omega(q)$ are now fully defined by its value.

\section{Numerical approximations and algorithm}

In the framework of finite-element method, we consider a discretized displacement field $\mathbf{U}$ together with consistent mass $\mathbf{M}$ and stiffness $\mathbf{K}$ structural matrices based on (5). Contact constraints (1) can be re-written as:

$$
\mathbf{g}(\mathbf{U})=\mathbf{A} \mathbf{U}-\mathbf{g}_{0}
$$

where rectangular matrix $\mathbf{A}$ restricts the displacement vector $\mathbf{U}$ to contact degrees-of-freedom.

Furthermore, a uniform time discretization is introduced:

$$
t=\left\{t_{k}=k T / m, k=1, \ldots, m\right\}
$$

such as $\overline{\mathbf{u}}_{k}=\mathbf{u}\left(t_{k}\right)$ are discrete time values of $\mathbf{u}$ and the Fourier series of Eq. (6) is truncated up to $N$-th order. Hence, frequency-domain and time-domain variables are now related to each other in a discrete form:

$$
\hat{\mathbf{u}}_{n}=\frac{1}{T} \sum_{k=1}^{m} \overline{\mathbf{u}}_{k} e^{-\mathrm{j} \frac{2 \pi k n}{m}} \quad \text { and } \quad \overline{\mathbf{u}}_{k}=\sum_{n=-N}^{N} \hat{\mathbf{u}}_{n} e^{\mathrm{j} \frac{2 \pi k n}{m}}
$$

In practical applications, it is usually required to have $N$ significantly smaller than $m$. 
The final eigenvalue problem is obtained from Eq. (15) in which space, time and frequency approximations are introduced and then reduces to $2 N+1$ coupled sub-problems:

$$
2 \mathbf{M}^{-1}\left(\mathbf{K}-(n \omega)^{2} \mathbf{M}\right) \hat{\mathbf{U}}_{n}+\mathbf{A}^{T} \sum_{k=1}^{m} \kappa\left(\mathbf{g}(\hat{\mathbf{U}})+\theta_{k}\right)_{+} e^{\mathrm{j} \frac{2 \pi k n}{m}}=0
$$

The proposed algorithm[1] based on the one detailed in [19] also features Powell's measure of convergence:

$$
G=\max _{\text {dof }}\left|\max _{k}\left(\mathbf{g}\left(\overline{\mathbf{u}}_{k}\right),-\theta_{k}\right)\right|
$$

and an adaptive procedure for updating penalty parameters and Lagrange multipliers.

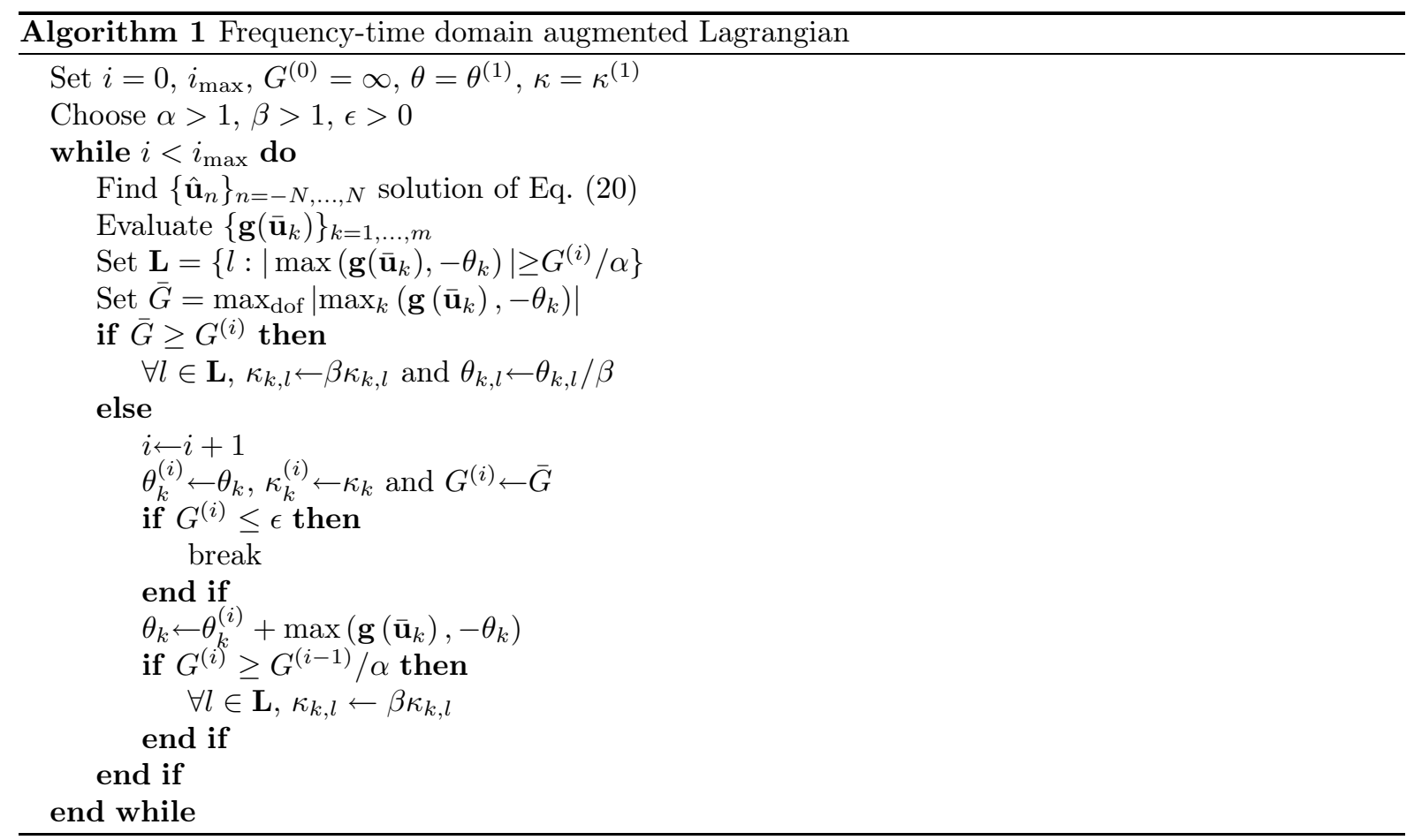

\section{Analysis of contact in compressor blades}

As an illustration of the proposed technique, we consider a turbomachinery compressor blade with a rigid contact interface at its tip edge. The finite element model of this blade is shown in Fig. 2. nodes in red define the contact interface. This model is reduced by means of a Craig-Bampton procedure composed of 72 constraints modes associated with displacements of 24 retained nodes belonging to contact interface and 40 component normal modes. Contact occurs in the radial direction only and a uniform initial gap is assumed. In the sequel, all displacements and velocities are normalized with respect to this initial gap and nonlinear frequencies are normalized with respect to the first linear frequency of the blade.

Figs. 3 display modal parameters of the first nonlinear mode (associated with first bending mode of the blade). In Fig. 3a, which represents the evolution of eigenfrequency as function of modal amplitude, two particular regions with distinct dynamical behaviors can be distinguished. The first refers to modal amplitude values such as the gap is always open and is characterized by a linear dynamics and constant frequency. The second, when the modal amplitude is such that the contact can occur at the interface, is highly nonlinear. The frequency-dependent amplitude phenomenon with stiffening effects is retrieved. Fig. $3 \mathrm{~b}$ shows a manifold for this mode, that is the displacement of one nonlinear coordinate plotted versus the displacement and velocity of a linear one (the master coordinate here). The effect of contact, which limits the amplitude of the nonlinear coordinate, is noticeable. 


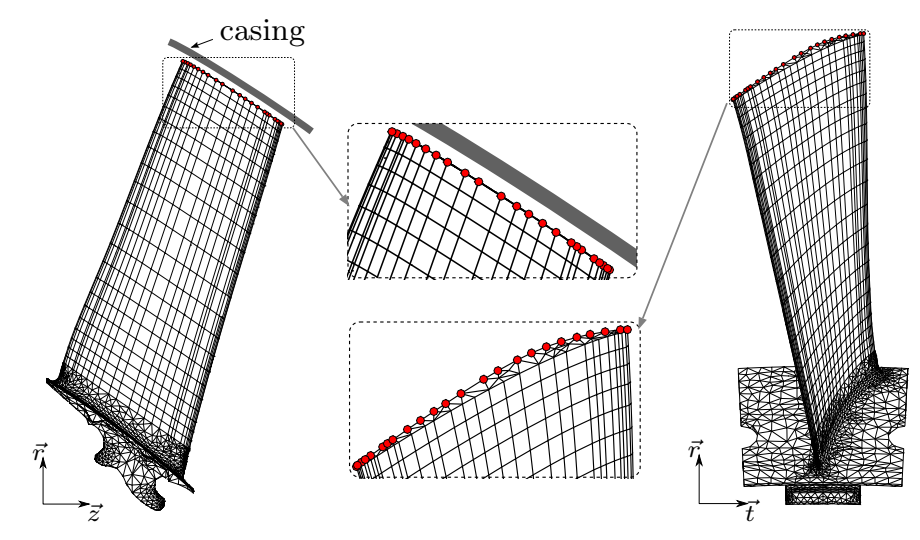

Figure 2: Blade model and details of the contact interface

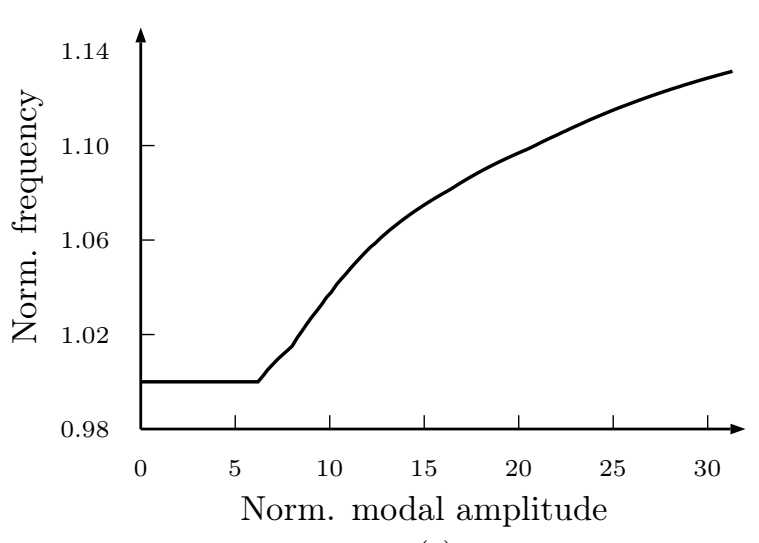

(a)

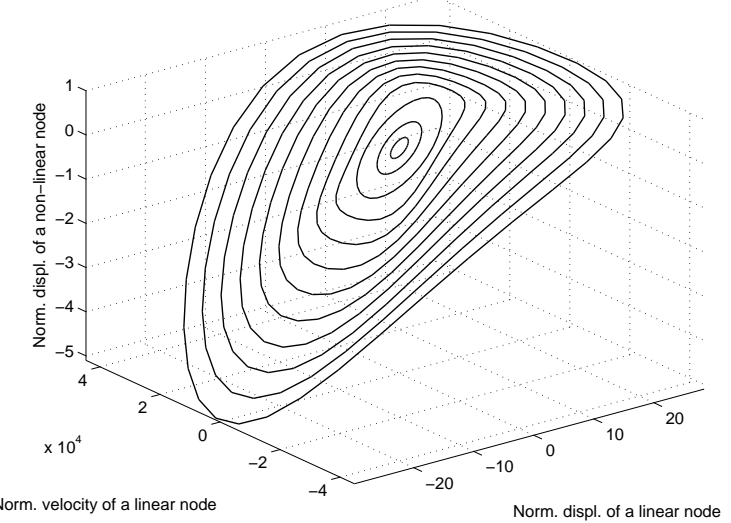

(b)

Figure 3: Modal parameters of the first nonlinear mode: (a) eigen-frequency / modal amplitude curve and (b) manifold.

In order to investigate in more details the effects of contact on modal parameters, Figs. 4 display the evolution of radial displacements of all edge nodes as function of time (one period) and for three selected values of the modal amplitude (with respect to Fig. 3a). Note that contact occurs when the radial displacements reach a unit value due to the chosen normalization. In Fig. 4a the modal amplitude has not reached the limit contact amplitude yet and no contact occurs. For the amplitude considered in Fig. $4 \mathrm{~b}$ contact occurs on the two last two nodes of the edge and during about $15 \%$ of time in one period. In the largest modal amplitude depicted Fig. 4c contact occurs on the last 4 nodes and also on the first 5 nodes of the edge and these two contact region are out of phase. These observations bring further explanations to the stiffening effects of contact which was noticed on Fig. 3a Indeed, stiffening is related to both the size of the actual contact interface (i.e. the number of nodes for which contact occurs) and the duration of contact.

Finally, Figs. [5 display the mode shapes on the finite element mesh for the three previous modal amplitude values. The changes in shape due to contact are substantial in particular during initial times of the period and can result in stress concentrations.

\section{Conclusions}

A methodology for modal analysis of elastic structures with contact interfaces was proposed. Based on the definition of nonlinear modes as periodic solutions of the autonomous dynamical system, the 


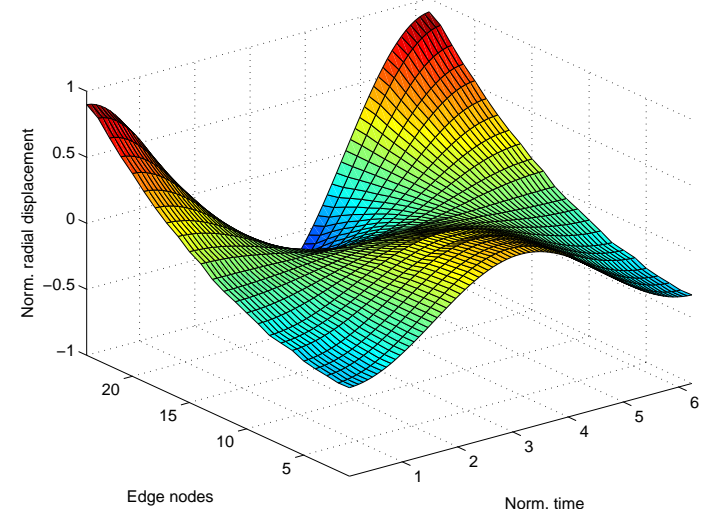

(a)

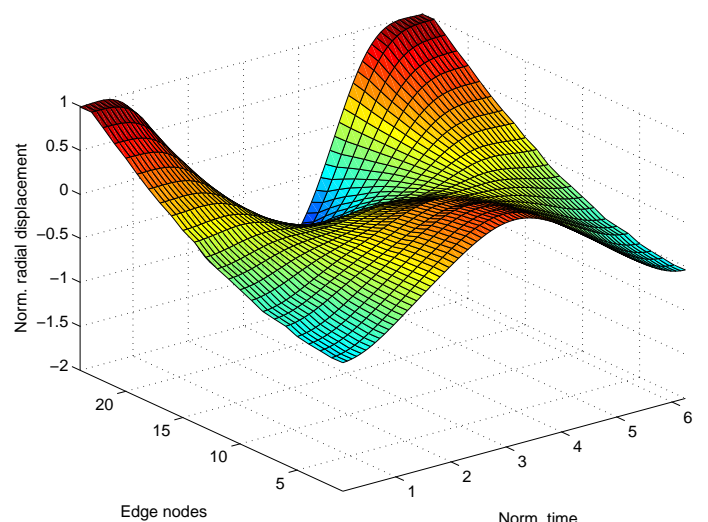

(b)

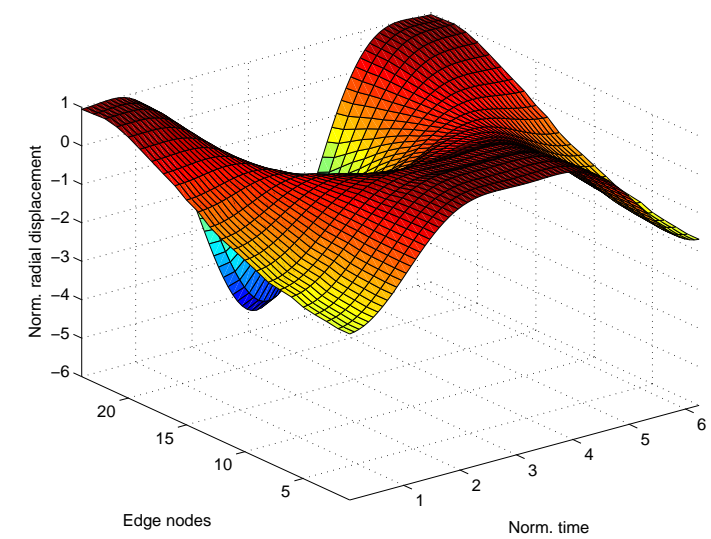

(c)

Figure 4: Radial displacements of edge nodes for three modal amplitudes: (a) 5.7, (b) 9.3 and (c) 31.3

eigenvalue problem is defined in a continuum mechanics framework with Signorini boundary conditions. A generalized Rayleigh quotient functional is introduced, which minimization under constraints is formulated using Lagrange multipliers and solved using an augmented Lagrangian approach. Numerical approximations in space, time and frequency are then introduced for the sake of numerical treatments.

As an application, a turbomachinery compressor blade in contact with a rigid casing is considered. The first nonlinear mode is studied using the proposed approach and the influence of contact (which depends on the amplitude of the motion) is investigated on modal parameters such as frequency and shapes.

Based on these encouraging developments, future works will focus on further investigations on nonlinear dynamics to include stability and bifurcations analysis and also more complex systems which include several deformable bodies in respective contacts. This tool can be used to predict dangerous interactions zones between blades and surrounding casings. In the future, friction effects and as well as wear of contact interfaces can also be studied.

\section{Acknowledgment}

Thanks go to Snecma for its technical and financial support. This work takes place in the framework of the MAIA mechanical research and technology program sponsored by CNRS, ONERA and SAFRAN Group. 


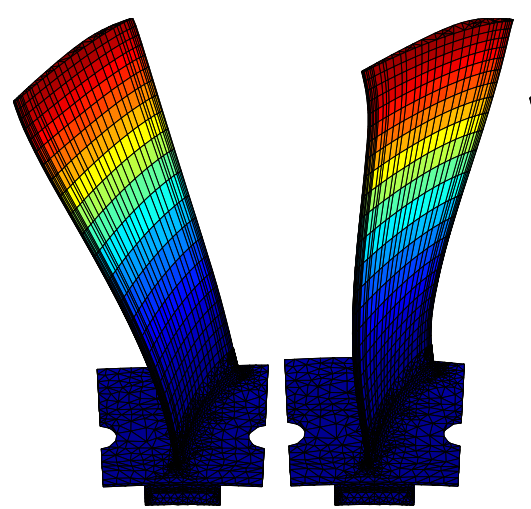

(a)

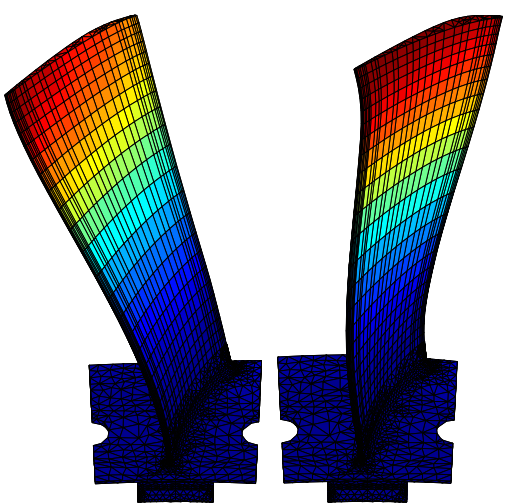

(b)

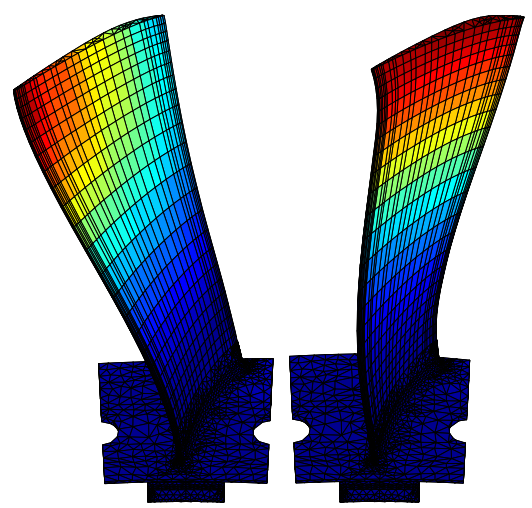

(c)

Figure 5: Mode shapes for three modal amplitudes: (a) 5.7, (b) 9.3 and (c) 31.3 and at $t=0$ and $t=T / 2$.

\section{References}

[1] A. F. Vakakis, L. I. Manevitch, Y. V. Mikhlin, V. N. Pilichuk, and A. A. Zevin. Normal Modes and Localization in Nonlinear Systems. Wiley series in nonlinear science, 1996.

[2] G. Kerschen, M. Peeters, J. C. Golinval, and A. F. Vakakis. Nonlinear normal modes, part I: A useful framework for the structural dynamicist. Mechanical Systems and Signal Processing, 23(1):170-194, 2009.

[3] E. Pesheck, C. Pierre, and S. W. Shaw. A new galerkin-based approach for accurate non-linear normal modes through invariant manifolds. Journal of Sound and Vibration, 249(5):971-993, 2002.

[4] R. Arquier, S. Bellizzi, R. Bouc, and B. Cochelin. Two methods for the computation of nonlinear modes of vibrating systems at large amplitudes. Computers \&5 Structures, 84(24-25):1565-1576, 2006.

[5] M. Peeters, R. Viguié, G. Sérandour, G. Kerschen, and J.-C. Golinval. Nonlinear normal modes, part II: Toward a practical computation using numerical continuation techniques. Mechanical Systems and Signal Processing, 23(1):195-216, 2009.

[6] D. Laxalde and F. Thouverez. Complex non-linear modal analysis for mechanical systems: Application to turbomachinery bladings with friction interfaces. Journal of Sound and Vibration, 322:1009-1025, 2009.

[7] P Alart and Curnier A. A mixed formulation for frictional contact problems prone to newton like solution methods. Computer Methods in Applied Mechanics and Engineering, 92(3):353, 1991.

[8] A.R. Mijar. Review of formulations for elastostatic frictional contact problems. Structural and Multidisciplinary Optimization, 20(3):167, 2000.

[9] N. J. Carpenter. Lagrange constraints for transient finite element surface contact. International Journal for Numerical Methods in Engineering, 32(1):103, 1991.

[10] T. A. Laursen and J. C. Simo. A continuum-based finite element formulation for the implicit solution of multibody, large deformation-frictional contact problems. International Journal for Numerical Methods in Engineering, 36(20):3451-3485, 1993.

[11] G. Pietrzak and A. Curnier. Large deformation frictional contact mechanics: continuum formulation and augmented lagrangian treatment. Computer Methods in Applied Mechanics and Engineering, 177(3-4):351, 1999. 
[12] K. C. Woo, A. A. Rodger, R. D. Neilson, and M. Wiercigroch. Application of the harmonic balance method to ground moling machines operating in periodic regimes. Chaos, Solitons 83 Fractals, 11(15):2515-2525, 2000.

[13] N. Leib, S. Nacivet, F. Thouverez, and L. Jézéquel. Experimental and numerical analysis of two vibro-impacting beams. In Proceedings of International Modal Analysis Conference, 2007.

[14] M. F. A. Azeez and A. F. Vakakis. Numerical and experimental analysis of a continuous overhung rotor undergoing vibro-impacts. International Journal of Non-Linear Mechanics, 34(3):415-435, 1998.

[15] N. Lesaffre, J.-J. Sinou, and F. Thouverez. Stability analysis of rotating beams rubbing on an elastic circular structure. Journal of Sound and Vibration, 299(4-5):1005-1032, 2007.

[16] M Legrand, C. Pierre, P. Cartraud, and J.-P. Lombard. Two-dimensional modeling of an aircraft engine structural bladed disk-casing modal interaction. Journal of Sound and Vibration, 319(12):366-391, 2009.

[17] P. Wriggers and G. Zavarise. Application of augmented lagrangian techniques for non-linear constitutive laws in contact interfaces. Communications in Numerical Methods in Engineering, 9(10):815824, 1993.

[18] M. J. D. Powell. Algorithms for nonlinear constraints that use lagrangian functions. Mathematical Programming, 14(1):224-248, 1978. 10.1007/BF01588967.

[19] J. S. Arora, A. I. Chahande, and J. K. Paeng. Multiplier methods for engineering optimization. International Journal for Numerical Methods in Engineering, 32(7):1485, 1991. 